\title{
Correlation between Collateral Ventilation and Interlobar Lung Fissures
}

\author{
Daniele Diso $^{a}$ Marco Anile $^{a}$ Carolina Carillo $^{a}$ Franco Ruberto $^{a}$ \\ Miriam Patella ${ }^{a}$ Emanuele Russo $^{\text {a }}$ Francesco Fraioli $^{b}$ Tiziano De Giacomo ${ }^{a}$ \\ Sara Mantovani ${ }^{a}$ Erino Rendina ${ }^{c}$ Federico Venuta ${ }^{c}$ \\ Departments of ${ }^{\mathrm{a}}$ Thoracic Surgery and ${ }^{\mathrm{b}}$ Radiology, Policlinico Umberto I, University of Rome Sapienza, and \\ ${ }^{\mathrm{c}}$ Fondazione Eleonora Lorillard Spencer Cenci, Rome, Italy
}

\section{Key Words}

Airway bypass - Bronchoscopic lung volume reduction .

Collateral lung ventilation $\cdot$ Emphysema $\cdot$ Interlobar fissures

\begin{abstract}
Background: New bronchoscopic techniques for end-stage emphysema treatment are nowadays available; the presence of interlobar collateral ventilation (CV) and interlobar lung fissures (ILF) is crucial for patient selection. Objectives: Assessment of these variables has been reported previously, but it has never been anatomically validated in vivo. This is the purpose of our study. Methods: Twenty-one patients undergoing lung resection for lung cancer were prospectively enrolled in this study. At operation, CV was assessed by the Chartis ${ }^{\circledR}$ catheter system. ILF completeness at high-resolution computed tomography (HRCT) was retrospectively reviewed. The ILF status at HRCT and at surgery was compared; furthermore, the relationship between CV and ILF status was assessed. Results: At HRCT, ILF were incomplete in 18 cases; at catheter evaluation, CV was present in 11 cases; 15 patients had incomplete ILF at operation. HRCT specificity, sensitivity and accuracy were 33,93 and $76 \%$ compared with ILF status at surgery. HRCT accuracy was $90 \%$ on the right and $63 \%$ on the left. We demonstrated a high grade of probabil-
\end{abstract}

ity of CV presence and incomplete ILF at surgery (odds ratio $=10.0$ ). Conclusions: There is a correlation between ILF status and CV. Both catheter evaluation of CV and HRCT assessment of ILF show some limitations. However, the cumulative information provided by these techniques allows to reliably assess the anatomical ILF status.

(c) 2014 S. Karger AG, Basel

\section{Introduction}

During the last decade, a number of bronchoscopic procedures have been proposed to improve respiratory mechanics and quality of life in patients with emphysema [1-9]. In particular, airway bypass approaches and endobronchial valve treatment (EBVT) are one step beyond in their clinical application. Collateral ventilation $(\mathrm{CV})$ plays a crucial albeit different role in the selection of patients.

$\mathrm{CV}$ is defined as 'the ventilation of alveolar structures through passages that bypass the normal airway' [10]. This happens through the pores of Kohn, the bronchioloalveolar communications of Lambert and the intrabronchiolar pathways of Martin. In emphysematous lungs, the progressive destruction of alveolar walls en-

\section{KARGER}

E-Mail karger@karger.com

www.karger.com/res
(C) 2014 S. Karger AG, Basel

0025-7931/14/0884-0315\$39.50/0
Federico Venuta, MD

Cattedra di Chirurgia Toracica, Policlinico Umberto I, Università di Roma Sapienza Fondazione Eleonora Lorillard Spencer Cenci

Viale del Policlinico 155, IT-00161 Rome (Italy)

E-Mail federico.venuta@uniromal.it 
larges these accessory pathways and bronchial obstruction increases expiratory resistance through the normal airway; this allows gas to move freely from one lobule to the other (intralobar CV); interlobar CV is also possible although it is obviously related to the absence of interlobar lung fissures (ILF) with consequent anatomical and functional communication between the lobes. Thus, incomplete ILF and interlobar CV are strictly related to one another.

On the base of this concept, Rendina et al. [4] hypothesized that the creation of extra-anatomic passages (airway bypass) between the proximal airway and the parenchyma in patients with homogeneous emphysema would bypass airway obstruction, decrease hyperinflation and improve lung mechanics. However, no sustainable benefit was recorded with this technique [11]. On the other side, EBVT should mimic lung volume reduction (LVR) surgery in patients with heterogeneous emphysema, where target areas can be clearly identified. In this setting, the worse emphysematous areas should have higher CV when compared to lobes with a density proximal to normal. Deployment of one-way endobronchial valves in the target emphysematous lobe favors progressive deflation of that area. Backward refilling of the 'valved' lobe should not exist if there is no interlobar CV. In this setting, ILF integrity is crucial since it should be a marker of the absence of interlobar CV. It has been demonstrated that EBVT results are improved by the presence of fissures and absence of interlobar CV $[8,12]$. For these reasons, assessment of ILF and CV is crucial to select the most appropriate procedure.

Completeness of ILF can be evaluated using high-resolution CT (HRCT) [13, 14]; however, radiological assessment may show some limitations and it has never been validated in vivo or at autopsy. On the other hand, several attempts to measure CV have been historically described, but they are all tedious particularly for patients with advanced emphysema and end-stage respiratory failure. A new minimally invasive method to measure CV has been recently proposed. The Chartis ${ }^{\circledR}$ system (Pulmonx, Redwood City, Calif., USA) [15-17] employs a flexible bronchoscopic catheter allowing indirect evaluation of interlobar CV through continuous measurement of air pressure and flow in an occluded lobe. However, also in this case, $\mathrm{CV}$ catheter measurements have never been validated by a correlation with the true anatomical ILF status.

In the present study, we have evaluated a group of patients undergoing lung resection; the presence/absence of $\mathrm{CV}$ measured using the Chartis system has been corre- lated with HRCT evidence of ILF; radiological and Chartis data have been subsequently validated by direct intraoperative assessment of ILF. So far, this is the first time that functional and radiological data have been anatomically validated in vivo. Although this is a purely anatomical and functional study, it could contribute to improve selection of patients for EBTV by including only patients with expected functional improvement.

\section{Patients and Methods}

The project was approved and partially sponsored by the University of Rome Sapienza (Prot. C26A10MLLM). All patients underwent the standard preoperative work-up including radiological (chest X-ray and HRCT), functional (pulmonary function tests and blood gas analysis) and cardiological (EKG and echocardiography when required) evaluation. Preoperative HRCT was retrospectively reviewed by a radiologist (F.F.); the presence of ILF was blindly determined using 3D reconstructions and multiplanar evaluation; ILF were defined as complete when at least $90 \%$ of the fissures were visible at HRCT.

In the operating room, after institution of standard monitoring, patients were intubated with a single-lumen endotracheal tube. Under spontaneous ventilation (topical 2\% lidocaine was added to avoid coughing), fiberoptic bronchoscopy was performed. Anesthesia was maintained with continuous infusion of propofol (6-10 $\mathrm{mg} / \mathrm{kg} / \mathrm{min}$ ) and alfentanil (50- to $100-\mu \mathrm{g}$ bolus titrated to maintain spontaneous ventilation). Through the operative channel of the fiberoptic bronchoscope, the Chartis catheter was advanced and placed under direct visual control in the lobar orifices. Pulmonx donated the catheters for study purposes. This is a hollow catheter shaft made of medical-grade Pebax. At the distal end of the catheter, there is an inflatable balloon; when inflated, it seals the airway so that air can flow out of the target compartment only through the catheter. The proximal end of the catheter has an obturator port; during expiration, the valve is open, allowing air to escape from the lung compartment; during inspiration, the valve is closed, preventing air from reentering the lung. Airflow and pressure exiting the catheter during spontaneous breathing are displayed on the console. In case of absent or insignificant interlobar $\mathrm{CV}$, the targeted lung compartment progressively contracts to the point that no expiratory flow can be recorded, reaching a steady state proximal or equal to zero. On the other hand, if interlobar $\mathrm{CV}$ is present, the expiratory air flow continues during recording, with a steady state almost equal to the preassessment level since the targeted lung is refilled by interlobar CV. On the left side, $\mathrm{CV}$ was assessed in the upper lobe; on the right side, it was measured in the 3 lobes.

After CV assessment, the fiberoptic bronchoscope is used rfor the correct placement of a bronchial blocker to enable lung ventilation during the surgical procedure; anesthesia is carried out as usual.

After entering the chest, the presence of fissures is visually assessed and recorded before performing lung resection. Patients with cancer extending to the ILF were excluded from the study. Fissures were considered complete when lobes were completely separated; limited incompleteness in the perihilar zone was considered as complete ILF; no quantification was performed. 
Quantitative variables were reported as means \pm SD; qualitative variables were reported as presence or absence of the event. ILF status at surgery was considered as the dependent variable and others as categorical covariates in univariate analysis performed by binary logistic regression (probit analysis). A value of $\mathrm{p}<0.05$ was considered statistically significant. Specificity, sensitivity and accuracy of HRCT and Chartis were calculated with Bayes indexes. The analysis was performed with SPSS software (version 17.0; SPSS Inc., Chicago, Ill., USA)

\section{Results}

Twenty-one patients (18 males and 3 females; mean age 67 years; range 54-79) scheduled for lung resection for cancer were prospectively enrolled in this study from October 2010 to April 2011. Sixteen were smokers. The mean $\mathrm{FEV}_{1}$ was $2.54 \pm 0.61 \mathrm{l} / \mathrm{s}$ (mean $90 \%$ predicted value; range $1.15-3.99 \mathrm{l} / \mathrm{s}$ ); 4 patients were in GOLD class 1 for emphysema $\left(\mathrm{FEV}_{1}<80 \%\right)$. Five patients received induction chemotherapy with a cis-platinum-based regimen. Tumor histology was squamous cell carcinoma in 11 cases, adenocarcinoma in 4 and metastatic from other sites in 6 . Ten patients were assessed on the right side and 11 on the left. We performed 11 lobectomies (4 right upper, 1 middle lobe, 3 left upper, 2 right lower and 2 left lower), 4 left pneumonectomies and 6 wedge resections ( 3 on the right side and 3 on the left). The results are reported in table 1 .

\section{HRCT Evaluation}

At HRCT, ILF were incomplete in 18 patients and complete in 3; of the 18 HRCT-visualized incomplete ILF, $14(77.8 \%)$ were confirmed at operation; in these 14 patients, catheter evaluation showed the presence of CV in 9 (64\%). There were 3 complete ILF on the left side and none on the right, and 8 incomplete ILF on the left and 10 on the right. Six patients were CV positive on the left side and 5 on the right; 5 patients were CV negative on the left and 5 on the right. Five patients had complete ILF at operation on the left and 1 on the right; 6 patients had incomplete ILF at operation on the left and 9 on the right side. Anatomically, incomplete ILF on the right side were located between the upper and middle lobe in 6 cases, between the upper and lower lobe in 1 and between the middle and lower lobe in 2.

\section{Evaluation}

Eleven patients were $\mathrm{CV}$ positive at catheter evaluation; in this group, 10 had incomplete and 1 had complete ILF at HRCT; the latter had incomplete ILF at operation.
Table 1. Summary of results

\begin{tabular}{|c|c|c|c|c|c|}
\hline $\begin{array}{l}\text { Patient } \\
\text { No. }\end{array}$ & Fissure CT & Fissure OR & $\mathrm{CV}$ & $\begin{array}{l}\text { Agree- } \\
\text { ment }\end{array}$ & Side \\
\hline 1 & incomplete & incomplete & present & yes & $\mathrm{L}$ \\
\hline 2 & incomplete & complete & absent & no & $\mathrm{L}$ \\
\hline 3 & incomplete & complete & absent & no & $\mathrm{R}$ \\
\hline 4 & incomplete & incomplete & present & yes & $\mathrm{R}$ \\
\hline 5 & incomplete & complete & present & no & $\mathrm{L}$ \\
\hline 6 & incomplete & incomplete & present & yes & $\mathrm{R}$ \\
\hline 7 & incomplete & incomplete & absent & no & $\mathrm{L}$ \\
\hline 8 & complete & complete & absent & yes & $\mathrm{L}$ \\
\hline 9 & incomplete & incomplete & present & yes & $\mathrm{R}$ \\
\hline 10 & incomplete & incomplete & present & yes & $\mathrm{R}$ \\
\hline 11 & incomplete & incomplete & present & yes & $\mathrm{L}$ \\
\hline 12 & incomplete & incomplete & absent & no & $\mathrm{R}$ \\
\hline 13 & incomplete & complete & absent & no & $\mathrm{L}$ \\
\hline 14 & incomplete & incomplete & absent & no & $\mathrm{L}$ \\
\hline 15 & incomplete & incomplete & absent & no & $\mathrm{R}$ \\
\hline 16 & incomplete & incomplete & absent & no & $\mathrm{R}$ \\
\hline 17 & incomplete & incomplete & present & yes & $\mathrm{R}$ \\
\hline 18 & complete & incomplete & present & no & $\mathrm{L}$ \\
\hline 19 & incomplete & incomplete & present & yes & $\mathrm{R}$ \\
\hline 20 & complete & complete & absent & yes & $\mathrm{L}$ \\
\hline 21 & incomplete & incomplete & present & yes & $\mathrm{L}$ \\
\hline
\end{tabular}

Fissure CT $=$ Visualization of fissures at CT; Fissure OR = visualization of fissures at surgery; $\mathrm{R}=$ right; $\mathrm{L}=$ left.

Ten patients were $\mathrm{CV}$ negative at catheter evaluation; 8 had incomplete ILF by HRCT and in 2 ILF were complete; 5 patients had incomplete ILF at operation and in 5 ILF were complete.

\section{Assessment at Operation}

Fifteen patients had incomplete ILF at operation: 14 of them had incomplete ILF at HRCT and 1 had complete ILF; 10 patients were CV positive and $5 \mathrm{CV}$ negative.

In the group of patients with complete ILF at operation, 4 had incomplete fissures at HRCT (1 CV positive). Five were CV negative and 1 was $C V$ positive.

Among the 4 patients with an $\mathrm{FEV}_{1}<80 \%(69 \pm 21 \%)$, 3 were evaluated and operated on the right side and 1 on the left. Three were CV positive and 1 was CV negative. All patients had incomplete ILF at HRCT. At operation, 2 ILF were complete and 2 were incomplete.

A summary of the aforementioned results is reported in table 1, and agreement between the three variables was considered: if there was a correspondence between HRCT, intraoperative visualization and $\mathrm{CV}$ evaluation, we reported yes, while in whatever other condition we indicated no. 
With respect to ILF status at surgery, HRCT specificity (true negative) was 33\%, sensitivity (true positive) was $93 \%$ and accuracy was $76 \%(16 / 21)$. Chartis catheter specificity was $83 \%$, sensitivity was $66.7 \%$ and accuracy was $71 \%(15 / 21)$. Chartis accuracy was $50 \%$ on the right side $(5 / 10)$ and $72 \%$ on the left (8/11). HRCT accuracy was $90 \%$ on the right $(9 / 10)$ and $63 \%$ on the left $(7 / 11)$. On the left side, a comparison between Chartis and HRCT evaluation showed no significant difference $(\mathrm{p}=0.4)$, and a comparison between HRCT and Chartis evaluation showed a trend towards significance $(\mathrm{p}=0.06)$.

The probit analysis showed a trend towards a statistically significant correlation between ILF status at surgery and CV evaluation $(\mathrm{p}=0.06)$, with a high probability of agreement between the presence of ILF and absence of $\mathrm{CV}$ (odds ratio $=10.0,95 \%$ confidence interval $=0.907$ 110.282), while ILF visualization at HRCT did apparently not correlate with CV $(\mathrm{p}=0.485)$. The predictability of HRCT regarding ILF status was of low grade ( $\mathrm{p}=$ 0.142 ; odds ratio $=0.143,95 \%$ confidence interval $=$ 0.01-2.012).

\section{Discussion}

The clinical application of new technologies usually follows better understanding of historical physiological concepts and the simplification of assessment of functional variables. CV and its implications in bronchoscopic LVR is a typical example of this strategy: the availability of modern technology has contributed to changes in the management of emphysema patients. As soon as it became evident that LVR surgery could be effective only in a well-selected group of patients, scientists and industries have promptly tried to minimize the risks of this procedures by developing new and less invasive approaches. Numerous attempts to achieve the same results with a bronchoscopic approach have been recently performed and several techniques have been proposed. In one way or the other, all these techniques rely on the presence or absence of interlobar $\mathrm{CV}$; thus, this variable became crucial in the patient selection process.

A number of techniques to assess CV have been previously employed, but no simple test to detect and quantify it has been reported. In vivo techniques include ${ }^{133} \mathrm{Xe}$ ventilation scintigraphy studies [18] and heliox measurements [19]. Other techniques involve placing the bronchoscope in a wedged position to allow insufflation of a constant gas flow through the working channel of the instrument to reach a steady-state pressure. At this time, the drop of pressure can be used to calculate the resistance to collateral flow [20].

Since the absence of interlobar CV was found to be strictly related to the presence of complete interlobar fissures, a number of studies have been reported to assess this anatomical entity at HRCT. Visual subjective criteria as well as automated computerized approaches [14] to quantitavely assess fissure integrity or completeness levels have been described. These systems carry a significative degree of reader-based limitations. Furthermore, they have never been anatomically validated by autoptic or operatory (thoracic surgery procedures) confirmation.

Our study is the first to evaluate the correlation between radiological assessment of ILF, interlobar CV and the true anatomical status; it demonstrates that both of them show a certain degree of inaccuracy. Overall, when catheter evaluation shows no CV, in most of the cases there are complete ILF and patients are certainly good candidates for EBVT; in these cases, atelectasis of the target lobe after valve placement can be predicted and excellent functional results can be anticipated. In case of absent ILF, there are other implications since catheter evaluation shows some limitations compared to the true anatomical findings. In particular, $50 \%$ of the $\mathrm{CV}$-negative patients had incomplete ILF at operation, although 5 of the 6 patients $(83.3 \%)$ with complete ILF at operation were CV negative; the only patient with incorrect catheter evaluation of complete ILF was also incorrectly assessed at HRCT. This is also true when incomplete ILF are observed at HRCT (only 78\% were confirmed at operation). In case of anatomically incomplete ILF, HRCT looks more adequate than catheter evaluation, especially on the right side; in fact, radiological work-up was correct in 14 of 15 patients (93\%); however, the only patient with incorrect HRCT assessment of incomplete ILF was correctly assessed by catheter evaluation (CV positive). Our statistical analysis showed a trend towards a significant correlation between CV and ILF intraoperative confirmation; furthermore, the high odds ratio outlined the strength of correlation between true anatomy and the presence of CV with respect to HRCT vision. Only prospective studies can confirm if the presence of interlobar CV is to be considered as a contraindication to valve placement or if it is simply a sign of very slow deflation of the target lobe.

One of the limitations of our study is related to the relatively high $\mathrm{FEV}_{1}$ of the study population since it includes only candidates scheduled for lung resection. However, if the Chartis system and HRCT are supposed to assess interlobar CV and ILF, respectively, this should be independent from the status of the lung parenchyma; 
assessment of the accuracy of a new technique in a normal environment is certainly the first step before validating it in patients with a specific disease. We can postulate that a higher grade of emphysema with a decreased $\mathrm{FEV}_{1}$ may produce different results in terms of CV and ILF radiological appearance, since the disease itself and repeated episodes of pulmonary and pleural infections may modi- fy these variables; however, this might be difficult to confirm since surgery, with the exception of LVR surgery, is rarely performed in this group of patients.

We can conclude that HRCT and catheter evaluation together are helpful to assess ILF and CV and to select patients for EBTV. However, none of them looks significantly superior to the other.

\section{References}

1 Reilly J, Washko G, Pinto-Plata V, Velez E, Kenney L, Berger R, Celli B: Biological lung volume reduction. A new bronchoscopic therapy for advanced emphysema. Chest 2007;131:1108-1113.

- 2 Herth FJ, Eberhard RE, Gompelmann D, Slebos DJ, Ernst A: Bronchoscopic lung volume reduction with a dedicated coil: a clinical pilot study. Ther Adv Respir Dis 2010;4141: 225-231.

>3 Emery MJ, Eveland RL, Evelandt K, Couetil LL, Hildebrandt J, Swenson ER: Lung volume reduction by bronchoscopic administration of steam. Am J Respir Crit Care Med 2010; 182:1282-1291.

-4 Rendina EA, De Giacomo T, Venuta F, Coloni GF, Meyers BF, Patterson GA, Cooper JD: Feasibility and safety of the airway by-pass procedure for patients with emphysema. J Thorac Cardiovasc Surg 2003;125:12941299.

5 Venuta F, De Giacomo T, Rendina EA, Ciccone AM, Diso D, Perrone A, Parola D, Anile M, Coloni GF: Bronchoscopic lung volume reduction with one way valves in patients with heterogeneous emphysema. Ann Thorac Surg 2005;79:411-416.

6 Venuta F, Rendina EA, De Giacomo T, Anile M, Diso D, Andreetti C, Pugliese F, Coloni GF: Bronchoscopic procedures for emphysema treatment. Eur J Cardiothorac Surg 2006; 29:281-287.
7 Venuta F, Anile M, Diso D, Carillo C, De Giacomo T, D'Andrilli A, Fraioli F, Rendina EA, Coloni GF: Long-term follow-up after bronchoscopic lung volume reduction in patients with emphysema. Eur Respir J 2012;39:10841089.

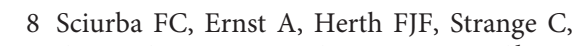
Criner GJ, Marquette CH, Kovitz KL, Chiacchierini RF, Goldin J, Mc Lennon G; Vent Study Research Group: A randomized study of endobronchial valves for advanced emphysema. N Engl J Med 2010;363:1233-1244.

-9 Venuta F, Diso D, Anile M, De Giacomo T, Rendina EA, Rolla M, Ricella C, Coloni GF: Bronchoscopic lung volume reduction as a bridge to lung transplantation in patients with chronic obstructive pulmonary disease. Eur J Cardiothorac Surg 2011;39:364-367.

10 Cetti EJ, Moore AJ, Geddes DM: Collateral ventilation. Thorax 2006;61:371-373.

11 Shah PL, Slebos DJ, Cardoso PFG, Cetti E, Voelker K, Levine B, Russell ME, Goldin J, Brown M, Cooper JD, Sybrecht GW; EASE trial study group: Bronchoscopic lung-volume reduction with Exhale airway stents for emphysema (EASE trial): randomized, shamcontrolled, multicenter trial. Lancet 2011;378: 997-1005.

12 Herth FGF, Eberhardt R, Gompelmann D, Ficker H, Wagner M, Ek L, Schmidt B, Slebos DJ: Radiological and clinical outcomes of using Chartis to plan endobronchial valve treatment. Eur Respir J 2013;41:302-308.

-13 Ozmen CA, Nazaroglu H, Bayrak AH, Senturk S, Akay HO: Evaluation of interlobar and accessory pulmonary fissures on 64-row MDTC. Clin Anat 2010;23:552-558.
$14 \mathrm{Pu}$ J, Fuhrman C, Durick J, Leader JK, Klym A, Sciurba FC, Gur D: Computerized assessment of pulmonary fissure integrity using high resolution CT. Med Phys 2010;37:46614672.

15 Aljury N, Freitag L: Validation and pilot study of a new bronchoscopic method to measure collateral ventilation before endobronchial lung volume reduction. J Appl Physiol 2009; 106:774-783.

-16 Gompelmann D, Eberhardt R, Michaud G, Ernst A, Herth FJ: Predicting atelectasis by assessment of collateral ventilation prior to endobronchial lung volume reduction: a feasibility study. Respiration 2010;80:419-425.

17 Mantri S, Macarag C, Shetty S, Aljury N, Herth F, Eberhardt R, Ernst A: Technical advances: measurement of collateral flow in the lung with a dedicated endobronchial catheter system. J Bronchology Interv Pulmonol 2009; 16:141-144.

18 Salanitri J, Kalff V, Kelly M, Halsworth L, Williams T, Snell G: ${ }^{13}$ Xenon ventilation scintigraphy applied to bronchoscopic lung volume reduction techniques for emphysema: relevance of interlobar collaterals. Intern Med J 2005;35:97-103.

19 Morrell NW, Wignall BK, Biggs T, Seed WA: Collateral ventilation and gas exchange in emphysema. Am J Respir Crit Care Med 1994; 150:635-643.

20 Terry PB, Traystman RJ, Neball HH: Collateral ventilation in man. N Engl J Med 1978; 298:10-15. 